\title{
Characterizing the conservation effect of clear coatings on photodegradation of wood
}

\author{
Pai-Lung Chou, Hui-Ting Chang, Ting-Feng Yeh, Shang-Tzen Chang * \\ School of Forestry and Resource Conservation, National Taiwan University, Taipei 106, Taiwan
}

Received 23 December 2006; received in revised form 24 February 2007; accepted 25 February 2007

Available online 18 April 2007

\begin{abstract}
Photodiscoloration of clear-coated wood may be caused by the yellowing of both clear coating film and underlying wood, or either of them. Wood specimens covered with two types of free polyurethane films with/without light stabilizer were used to simulate the photodiscoloration of clear-coated wood. Percent UV transmission of aromatic polyurethane (PU) films decreased after irradiation, whereas aliphatic polyurethane (PUA) films significantly increased with irradiation time resulting in further photoyellowing of wood beneath the PUA film. A light reflection model was used to elucidate discoloration caused separately by the clear coating film and the underlying wood. After 24 days of light irradiation, clear coating and the underlying wood contributed respectively, 40.70\% and 59.30\% discoloration of PU-coated specimens, and the corresponding values for PUA-coated specimens were 5.15\% and $94.85 \%$. PU film with light stabilizer reduced lignin degradation and generation of carbonyl derivatives in the underlying wood.
\end{abstract}

(c) 2007 Elsevier Ltd. All rights reserved.

Keywords: Wood; Polyurethane coating; Photodiscoloration; Free film; Lightfastness test

\section{Introduction}

Wood is mainly composed of cellulose, hemicelluloses, lignin, and extractives. It is a favorite indoor and outdoor constructions material because of its abundance, attractive appearance and easy processing. However, unprotected wood is susceptible to discoloration and deterioration that reduce its mechanical and physical properties (Hon, 2001; Pastore et al., 2004; Williams, 2005). Many methods, such as surface coating, chemical modification and impregnation with chemicals or plastics have been found to be able to extend service life of wood (Deka and Saikia, 2000; Gindl et al., 2003; Barnes et al., 2005; Chang and Chang, 2006). Among these methods, surface coating is the most common way to protect wood against degradation and to enhance its distinctive appearance (Hayoz et al., 2003; Buckle et al., 2005). Therefore, characteristics of wood

\footnotetext{
* Corresponding author. Tel.: +88623366 4626; fax: +886223654520. E-mail address: peter@ntu.edu.tw (S.-T. Chang).
}

coatings to withstand mechanical or chemical abrasion and to tolerate expansion and contraction of wood are critical to prolong their protective functions (Schwalm et al., 1997).

Polyurethane polymers are considered to be high-quality coatings. In the coating industry, two-component solvent-based polyurethane systems have been widely used because desirable properties can be obtained by adjusting makeup components (Lai and Quinn, 1995; Kim and Pail, 1999). Polyurethane coatings provide not only room temperature cure response, but also excellent abrasion resistance, high strength, hardness, flexibility, adhesion to wood materials, great modulus with elongation at break, and good chemical resistance (Fiori, 1997; Kultys and Pikus, 2001). However, wood coated with clear polyurethane coating is still susceptible to photoyellowing (Sigh et al., 2001). Photodiscoloration is unavoidable even if wood is coated with non-yellowing or durable clear coatings, such as aliphatic polyurethane coatings (Chang and Chou, 1999). Yellowing of both clear coating and underlying wood, or either of them may cause the discoloration. 
In the CIE $L^{*} a^{*} b^{*}$ system, discoloration of a specimen after light irradiation is described by color difference $\left(\Delta E^{*}\right)$. However, color difference contributed by clear coating or underlying wood can not be proportioned separately from a single $\Delta E^{*}$ value in the coated wood system.

Objectives of this study were to study photostability properties of coated wood systems and to investigate ways to improve polyurethane-coated wood against photodiscoloration. Photodiscoloration of coated wood was simulated by wood specimens covered with different free polyurethane films with/without a commercial light stabilizer (Tinuvin-1130). A light reflection model based on color factors (tristimulus values) $X, Y$, and $Z$ was established in order to quantify proportion of discoloration caused by clear coating and underlying wood separately. The model was employed to discuss the efficacy of different clear coating treatments. Chemical changes of wood under different films were also characterized after lightfastness test.

\section{Methods}

\subsection{Wood specimens and formulation of coatings}

China fir (Cunninghamia lanceolata) wood $50 \mathrm{~mm} \times$ $75 \mathrm{~mm} \times 10 \mathrm{~mm}$ in dimension was used in this study.

Commercial aromatic polyurethane (PU) and aliphatic polyurethane (PUA) (Lignal Co., Germany) with a solid content ca. $51 \%$ and $27 \%$, respectively, were used in this study. Also used were PU and PUA containing 2\% Tinuvin-1130 (hydroxyphenyl benzotriazole light absorber) based on solid content of the clear coatings, which were respectively labeled as PUS and PUAS.

\subsection{Preparation of polyurethane-coated wood and free films}

Wood specimens were coated with PU and PUA coatings with or without $2 \%$ light stabilizer. The thickness of the wet coatings applied onto wood surfaces was ca. $200 \mu \mathrm{m}$. For preparation of free films, wet clear coatings were applied onto glass surfaces at a thickness approximately $200 \mu \mathrm{m}$, followed by curing at the ambient temperature and peeling off the cured films for later experiments.

\subsection{Lightfastness tests and color measurements}

A Q-panel QUV accelerated lightfastness tester with eight UVA-351 lamps was used for accelerated lightfastness test. Specimens were exposed to UVA-351 lamps directly. The temperature of the black panel was $60 \pm 2{ }^{\circ} \mathrm{C}$.

The color parameters used in this study were calculated from spectral reflectance curves obtained with a diffuse reflectance UV/Vis spectrophotometer (Jasco V550) equipped with an integrating sphere attachment. The tristimulus values $(X, Y$, and $Z$ ) of the coated wood, free films and wood underneath were calculated from spectral reflectance curves directly. The $L^{*}, a^{*}, b^{*}, \Delta E^{*}$ (color difference) and $\Delta \mathrm{YI}$ (difference of yellowness index) color parameters were then computed using the CIE (Commission Internationale de l'Eclairage, 1976) $L^{*} a^{*} b^{*}$ equation.

\subsection{Models for elucidating discoloration of clear-coated wood samples}

In the CIE $L^{*} a^{*} b^{*}$ system discoloration of a specimen after irradiation is described by color difference $\left(\Delta E^{*}\right)$. However, discoloration contributed by clear coating and underlying wood cannot be proportioned directly from a single $\Delta E^{*}$ value in coated wood system. In order to do that, wood specimens were covered tightly with dry free coating films using clamps to simulate coated wood. After irradiated at various durations color changes of the free coating films and the underlying wood were measured separately, and the results were used to elucidate photodiscoloration in clear-coated wood. In the CIE $L^{*} a^{*} b^{*}$ system, the tristimulus values for a reflecting sample are calculated by adding the product of the spectral power distribution of the illuminant (Marcus, 1998). A model described below was used to clarify the tristimulus reflected by the coating and wood.

In this model (Fig. 1a) the original tristimulus $\left(X_{0}, Y_{0}, Z_{0}\right)$ emitted from light source $\left(D_{65}\right)$ is divided into two portions: the partial tristimulus absorbed by free film $\left(A_{\mathrm{f}_{1}}+A_{\mathrm{f}_{2}}+\right.$ $\left.A_{\mathrm{f}_{3}}+A_{\mathrm{f}_{4}} \ldots\right)$ and the others reflected by standard white board $\left(E_{\mathrm{r}_{1}}\right)$. The total reflected tristimulus $\left(E_{\mathrm{t}}\right)$ of free film is obtained by UV/Vis spectrometer. Since the absorption of film is not uniform in the visible region, the true absorption of film (film factor, $T_{\mathrm{f}}$ ) of $X_{0}, Y_{0}, Z_{0}$ was calculated by the following equations separately. In this model $E_{\mathrm{t}}$ is a geometric series, and the ratio $r_{\mathrm{f}}$ is expressed below:

$$
\begin{aligned}
E_{\mathrm{t}_{1}}= & \left(E_{0}-E_{\mathrm{r}_{1}}\right) \times T_{\mathrm{f}} \\
E_{\mathrm{t}_{2}}= & E_{\mathrm{t}_{1}} \times\left(1-\frac{E_{\mathrm{r}_{1}}}{E_{0}}\right) \times T_{\mathrm{f}} \\
E_{\mathrm{r}_{2}}= & E_{\mathrm{t}_{1}} \times \frac{E_{\mathrm{r}_{1}}}{E_{0}} \\
E_{\mathrm{t}_{3}}= & E_{\mathrm{r}_{2}} \times\left(1-\frac{E_{\mathrm{r}_{1}}}{E_{0}}\right) \times T_{\mathrm{f}} \\
E_{\mathrm{t}}= & E_{\mathrm{r}_{1}}+E_{\mathrm{t}_{2}}+E_{\mathrm{t}_{3}}+E_{\mathrm{t}_{4}}+\cdots \\
= & E_{\mathrm{r}_{1}}+E_{0} \times\left(1-\frac{E_{\mathrm{r}_{1}}}{E_{0}}\right)^{2} \times T_{\mathrm{f}}^{2}+E_{0} \\
& \times\left(1-\frac{E_{\mathrm{r}_{1}}}{E_{0}}\right)^{2} \times T_{\mathrm{f}}^{2} \times \frac{E_{\mathrm{r}_{1}}}{E_{0}} \times\left(1-\frac{E_{\mathrm{r}_{1}}}{E_{0}}\right)^{2} \\
& +E_{0} \times\left(1-\frac{E_{\mathrm{r}_{1}}}{E_{0}}\right)^{2} \times T_{\mathrm{f}}^{2} \times\left(\frac{E_{\mathrm{r}_{1}}}{E_{0}}\right)^{2} \times\left(1-\frac{E_{\mathrm{r}_{1}}}{E_{0}}\right)^{2}+\cdots \\
r_{\mathrm{f}}= & \frac{E_{\mathrm{r}_{1}} \times\left(1-\frac{E_{\mathrm{r}_{1}}}{E_{0}}\right)^{2}}{E_{0}}= \\
E_{\mathrm{t}}= & E_{\mathrm{r}_{1}}+E_{0} \times\left(1-\frac{E_{\mathrm{r}_{1}}}{E_{0}}\right)^{2} \times T_{\mathrm{f}}^{2} \times\left(\frac{1}{1-r_{\mathrm{f}}}\right)^{2} \times\left(1-\frac{E_{\mathrm{r}_{1}}}{E_{0}}\right)^{2} \\
\Rightarrow & T_{\mathrm{f}}=\sqrt{\frac{\left(E_{\mathrm{t}}-E_{\mathrm{r}_{1}}\right) \times\left(1-r_{\mathrm{f}}\right)}{E_{0}}}(1)
\end{aligned}
$$


a
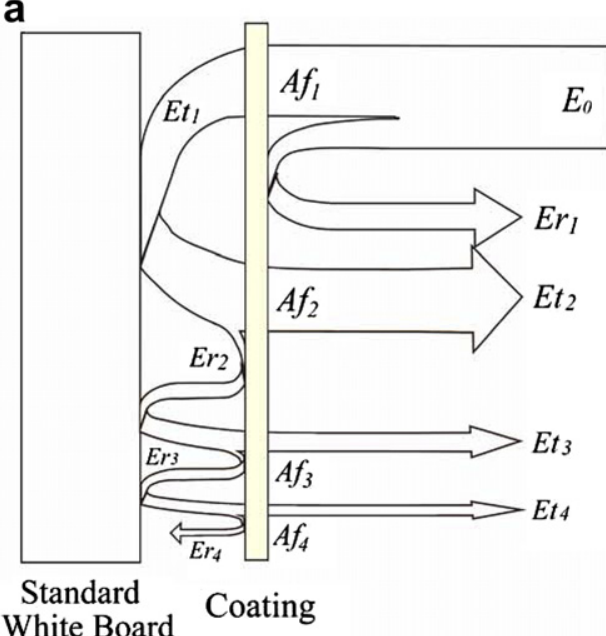

Standard

White Board

\section{b}

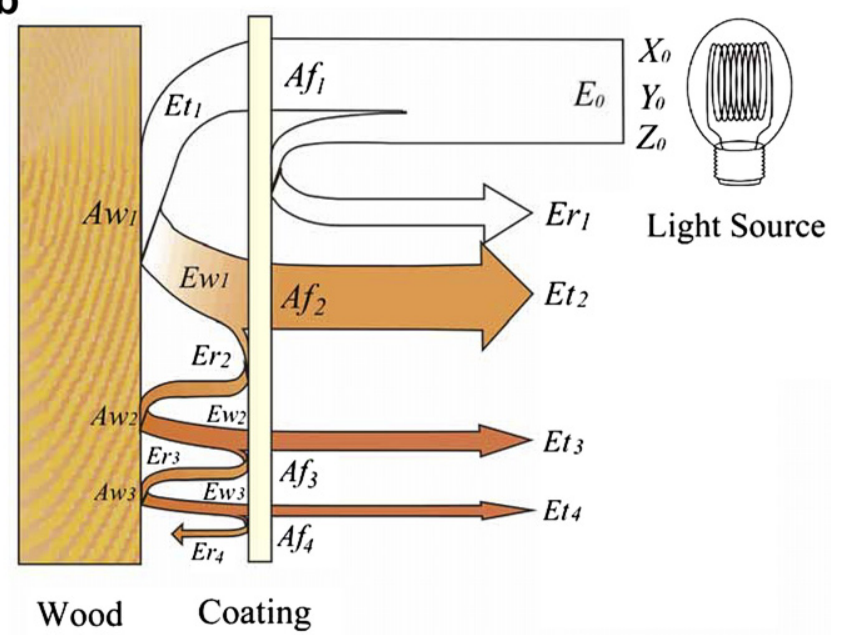

Fig. 1. Light reflection model. (a) Free coating film; (b) wood covered with free coating film.

where $E_{0}$ is intensity emitted from light source, $E_{\mathrm{r}_{n}}$ is intensities reflected from coating surfaces with $n=1,2,3, \ldots$ and $E_{\mathrm{t}_{n}}$ is intensities transmitted through coating films with $n=1,2,3, \ldots$

The tristimulus observed in UV/Vis spectrometer could be distributed among the coating surface and underlying wood as shown in Fig. 1b. $E_{\mathrm{w}_{n}}$ : intensities reflected from wood, $n=1,2,3, \ldots ; T_{\mathrm{f}}$ : film factor, the absorption of film; $A_{\mathrm{f}_{n}}$ : intensities absorbed by film, $n=1,2,3, \ldots ; A_{\mathrm{w}_{n}}$ : intensities absorbed by wood, $n=1,2,3, \ldots$.

The percentage of tristimulus from wood and coating can be calculated by the following equations. In this model, the series of $E t_{2}+E t_{3}+E t_{4}, \ldots$ is a geometric series, and the ratio $r_{\mathrm{w}}$ is expressed as in (2). The $\Delta E^{*}$ values attributed to coating and wood were portioned by the percentage changes of tristimulus after lightfastness test.

$E_{0}=X_{0} ; Y_{0} ; Z_{0}$

Reflection from free film:

$E_{\mathrm{r}_{n}}=X_{\mathrm{r}_{n}} ; \quad Y_{\mathrm{r}_{n}} ; Z_{\mathrm{r}_{n}}, n=1,2,3, \ldots$
Reflection from wood:

$$
\begin{aligned}
X_{\mathrm{w}}= & E_{\mathrm{t}_{2 x}}+E_{\mathrm{t}_{3 x}}+E_{\mathrm{t}_{4 x}}+\cdots ; \\
Y_{\mathrm{w}}= & E_{\mathrm{t}_{2 y}}+E_{\mathrm{t}_{3 y}}+E_{\mathrm{t}_{4 y}}+\cdots ; \\
Z_{\mathrm{w}}= & E_{\mathrm{t}_{2 z}}+E_{\mathrm{t}_{z z}}+E_{\mathrm{t}_{4 z}}+\cdots ; \\
E_{\mathrm{t}_{2}}+ & E_{\mathrm{t}_{3}}+E_{\mathrm{t}_{4}}+\cdots \\
= & E_{\mathrm{w}} \times\left(1-\frac{E_{\mathrm{r}_{1}}}{E_{0}}\right)^{2} \times T_{\mathrm{f}}^{2}+E_{\mathrm{w}} \times\left(1-\frac{E_{\mathrm{r}_{1}}}{E_{0}}\right)^{2} \times T_{\mathrm{f}}^{2} \\
& \times \frac{E_{\mathrm{r}_{1}}}{E_{0}} \times \frac{E_{\mathrm{w}}}{E_{0}} \times\left(1-\frac{E_{\mathrm{r}_{1}}}{E_{0}}\right)+E_{\mathrm{w}} \times\left(1-\frac{E_{\mathrm{r}_{1}}}{E_{0}}\right)^{2} \\
& \times T_{\mathrm{f}}^{2} \times \frac{E_{\mathrm{r}_{1}}}{E_{0}} \times\left(\frac{E_{\mathrm{w}}}{E_{0}}\right)^{2} \times\left(1-\frac{E_{\mathrm{r}_{1}}}{E_{0}}\right)^{2}+\cdots \\
r_{\mathrm{w}}= & \frac{E_{\mathrm{r}_{1}} \times E_{\mathrm{w}}}{E_{0}^{2}} \times\left(1-\frac{E_{\mathrm{r}_{1}}}{E_{0}}\right) \\
E_{\mathrm{t}_{2}}+ & E_{\mathrm{t}_{3}}+E_{\mathrm{t}_{4}}+\cdots=E_{\mathrm{w}} \times\left(1-\frac{E_{\mathrm{r}_{1}}}{E_{0}}\right)^{2} \times T_{\mathrm{f}}^{2} \times\left(\frac{1}{1-r_{\mathrm{w}}}\right)
\end{aligned}
$$

Total reflection:

$X_{\mathrm{t}}=X_{\mathrm{f}}+X_{\mathrm{w}} ; \quad Y_{\mathrm{t}}=Y_{\mathrm{f}}+Y_{\mathrm{w}} ; \quad Z_{\mathrm{t}}=Z_{\mathrm{f}}+Z_{\mathrm{w}}$

Percentage of reflection from free film $\left(P_{\mathrm{f}}\right)$ :

$\left(X_{\mathrm{f}}+Y_{\mathrm{f}}+Z_{\mathrm{f}}\right) /\left(X_{\mathrm{t}}+Y_{\mathrm{t}}+Z_{\mathrm{t}}\right) \times 100$

Percentage of reflection from wood $\left(P_{\mathrm{w}}\right)$ :

$\left(X_{\mathrm{w}}+Y_{\mathrm{w}}+Z_{\mathrm{w}}\right) /\left(X_{\mathrm{t}}+Y_{\mathrm{t}}+Z_{\mathrm{t}}\right) \times 100$

Color difference value calculated for free film $\left(\Delta E_{\mathrm{f}}^{*}\right)$ :

$P_{\mathrm{f}} \times \Delta E_{\mathrm{af}}^{*}$

$\Delta E_{\mathrm{af}}^{*}$ is the color difference value measured from film in front of standard white board. Color difference value calculated for wood $\left(\Delta E_{\mathrm{w}}^{*}\right)$ :

$P \mathrm{~W} \times \Delta E_{\mathrm{aw}}^{*}$

$\Delta E_{\mathrm{aw}}^{*}$ is the color difference value measured from individual wood. Total calculated color difference value resulted from free film and wood $\left(\Delta E_{\text {total }}^{*}\right)$ :

$\Delta E_{\text {total }}^{*}=\Delta E_{\mathrm{f}}^{*}+\Delta E_{\mathrm{w}}^{*}$

Percentage of total discoloration caused by free film $\left(D_{\mathrm{f}}\right)$ :

$D_{\mathrm{f}}(\%)=\Delta E_{\mathrm{f}}^{*} / \Delta E_{\text {total }}^{*} \times 100$

Percentage of total discoloration caused by wood beneath free film $\left(D_{\mathrm{w}}\right)$

$D_{\mathrm{w}}(\%)=\Delta E_{\mathrm{w}}^{*} / \Delta E_{\mathrm{total}}^{*} \times 100$

\subsection{Spectral analyses}

A UV/Vis spectrophotometer (Jasco V-550) was used to measure UV/Vis transmission spectra of free polyurethane films. The scanning range was from 200 to $800 \mathrm{~nm}$. A FTIR (Bio-rad FTS-40) spectrometer equipped with a Spectra Tech diffuse reflectance accessory unit was used for moni- 
toring the chemical changes on wood surfaces. The spectral resolution was $4 \mathrm{~cm}^{-1}$ over the range of $400-4000 \mathrm{~cm}^{-1}$.

\section{Results and discussion}

\subsection{Photodiscoloration of wood coated or covered with polyurethane films}

Color difference value $\left(\Delta E^{*}\right)$ of wood covered with free PU film (24.96) was similar to that of PU-coated wood (22.24) after 24 days of irradiation. The $\Delta E^{*}$ values of PUS-coated wood and wood covered with PUS film were almost identical (18.85 and 18.61). Difference in $\Delta E^{*}$ between PUAS-coated wood (16.93) and wood covered with PUAS film (13.71) was slightly greater but still was visually indistinguishable. Results obtained herein indicated that discoloration of wood covered with free films was approximately the same as that of coated wood, and thus this film/wood overlay system can be used to clarify the relationships between coating films and wood beneath the coating in photodiscoloration and photodegradation.

Measurements of discoloration of free films and wood beneath the free films are shown in Table 1. After 24 days of UV irradiation $\Delta E^{*}$ values of free films in descending order were PU (33.04), PUS (25.20), PUA (2.77), and PUAS (1.99). Addition of $2 \%$ Tinuvin-1130 into PU was

Table 1

Color differences $\left(\Delta E^{*}\right)$ and difference of yellowness index $(\Delta \mathrm{YI})$ of specimens after 24 days of UV irradiation

\begin{tabular}{lrr}
\hline Specimens & $\Delta E^{*}$ & $\Delta$ YI \\
\hline PU free film & 33.04 & 48.09 \\
Wood beneath the PU free film & 20.65 & 36.68 \\
PUS free film & 25.20 & 37.48 \\
Wood beneath the PUS free film & 12.69 & 23.28 \\
PUA free film & 2.77 & 1.64 \\
Wood beneath the PUA free film & 24.04 & 43.13 \\
PUAS free film & 1.99 & 2.33 \\
Wood beneath the PUAS free film & 19.46 & 34.99
\end{tabular}

PU: aromatic polyurethane; PUS: PU with $2 \%$ Tinuvin-1130; PUA: aliphatic polyurethane; PUAS: PUA with $2 \%$ Tinuvin- 1130 . more effective in reducing discoloration than adding it to PUA because PUA was formulated as a non-yellowing aliphatic polyurethane resin. $\Delta E^{*}$ values of wood after UV irradiation in descending order were that of the wood beneath PUA film (24.04), wood beneath PU film (20.65), wood beneath PUAS film (19.46), and wood beneath PUS film (12.69). Changes in yellowness index $(\Delta \mathrm{YI})$ of tested wood specimens was consistent with $\Delta E^{*}$ after 24 days of irradiation. Although PUA and PUAS were nonyellowing resin, discoloration of wood beneath PUA and PUAS films were surprisingly more severe than that beneath PU and PUS films. Addition of 2\% Tinuvin-1130 into PU apparently was effective in reducing discoloration of the underlying wood.

As shown in UV-Vis transmittance spectra of free films (Fig. 2), the transmitted UV wavelength range of PUA film was wider than that of PU film. Addition of the light stabilizer into both polyurethane films can effectively cut off the UV light. Percent UV transmittance of PU film decreased after irradiation. On the contrary, UV transmission of PUA film increased significantly with irradiation time, resulting in further photoyellowing of the wood beneath. The reduced UV transmission of PUS film provided effec-

Table 2

Photodiscoloration of simulated clear-coated wood system resulted from PU film and the underlying wood using the light reflection model

\begin{tabular}{lrrrrrr}
\hline Parameters $^{\mathrm{a}}$ & \multicolumn{7}{l}{ Irradiation time (days) } \\
\cline { 2 - 7 } & \multicolumn{1}{c}{2} & \multicolumn{1}{c}{4} & \multicolumn{1}{c}{8} & \multicolumn{1}{c}{12} & \multicolumn{1}{c}{16} & \multicolumn{1}{c}{24} \\
\hline$P_{\mathrm{f}}(\%)$ & 25.52 & 26.64 & 27.87 & 28.95 & 30.05 & 30.02 \\
$\Delta E_{\mathrm{af}}^{*}$ & 9.92 & 15.17 & 21.65 & 24.72 & 27.53 & 33.04 \\
$\Delta E_{\mathrm{f}}^{*}$ & 2.53 & 4.04 & 6.03 & 7.16 & 8.27 & 9.92 \\
$P_{\mathrm{w}}(\%)$ & 74.48 & 73.36 & 72.13 & 71.05 & 69.95 & 69.98 \\
$\Delta E_{\mathrm{aw}}^{*}$ & 10.55 & 13.47 & 15.19 & 16.76 & 18.62 & 20.65 \\
$\Delta E_{\mathrm{w}}^{*}$ & 7.86 & 9.88 & 10.96 & 11.91 & 13.02 & 14.45 \\
$\Delta E_{\mathrm{total}}^{*}$ calculated & 10.39 & 13.92 & 16.99 & 19.06 & 21.30 & 24.37 \\
$\Delta E^{*}$ measured & 11.75 & 15.45 & 19.09 & 21.14 & 22.81 & 24.96 \\
$D_{\mathrm{f}}(\%)$ & 24.37 & 29.03 & 35.51 & 37.54 & 38.84 & 40.70 \\
$D_{\mathrm{w}}(\%)$ & 75.63 & 70.97 & 64.49 & 62.46 & 61.16 & 59.30 \\
\hline
\end{tabular}

${ }^{\text {a }}$ See Section 2.4 .

b Directly from wood covered with film.
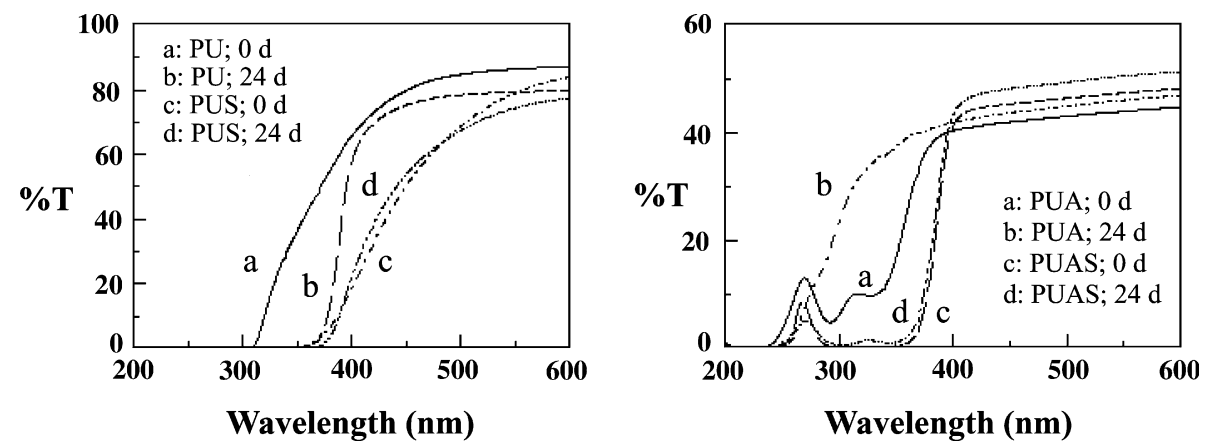

Fig. 2. UV-Vis transmission spectra of polyurethane free films before and after 24-day irradiation. PU: aromatic polyurethane; PUS: PU with $2 \%$ Tinuvin-1130; PUA: aliphatic polyurethane; PUAS: PUA with 2\% Tinuvin-1130. 
tive protection of the underlying wood against discoloration.

\subsection{Proportioned photodiscoloration model of clear-coated wood}

$\Delta E^{*}$ of free PU film (33.04) and $\Delta E^{*}$ of wood beneath $\mathrm{PU}$ film (20.65) together, as shown in Table 1, was much greater than the combined $\Delta E^{*}$ of wood covered with free PU film (24.96) or PU-coated wood (22.24). Therefore, it is necessary to use the light reflection model (Fig. 1) to quantify proportion of discoloration caused by clear coating and underlying wood. Based on the model, after 2 days of UV irradiation $\Delta E_{\mathrm{f}}^{*}$, which is color change contributed by free PU free and calculated by multiplying $P_{\mathrm{f}}(25.52 \%)$ and $\Delta E_{\mathrm{af}}^{*}(9.92)$, was found to be 2.53 (Table 2). Similarly, color change contributed from wood beneath PU film $\left(\Delta E_{\mathrm{w}}^{*}\right)$ was calculated to be 7.86. $\Delta E_{\text {total }}^{*}$, as calculated from $\Delta E_{\mathrm{f}}^{*}+\Delta E_{\mathrm{w}}^{*}=10.39$, was found to be close to the measured $\Delta E^{*}$ value (11.75) of PU-coated wood. Therefore, percent of discoloration caused by PU film $\left(D_{\mathrm{f}}\right)$ and the wood beneath PU film
$\left(D_{\mathrm{w}}\right)$ were $24.37 \%$ and $75.63 \%$, respectively. After 24 days of UV irradiation, the calculated $\Delta E_{\text {total }}^{*}$, value 24.37 also was close to the measured $\Delta E^{*}$ (24.96), and respective percent contributions of discoloration from PU film $\left(D_{\mathrm{f}}\right)$ and wood beneath PU film $\left(D_{\mathrm{w}}\right)$ were $40.70 \%$ and $59.30 \%$. Correlation between calculated $\Delta E_{\text {total }}^{*}$ and measured $\Delta E^{*}$ was estimated to be $y=1.08 x$ with $R^{2}=0.97$. Thus, discoloration contributed from the coating film and the underlying wood in the coated wood system could be quantified separately by using this light reflection model.

Fig. 3 shows changes in $\Delta E_{\mathrm{f}}^{*}, \Delta E_{\mathrm{w}}^{*}$, and $\Delta E_{\mathrm{total}}^{*}$, of specimens in the PU and PUS systems during UV irradiation for up to 24 days. $\Delta E_{\mathrm{f}}^{*}$ of PU film and $\Delta E_{\mathrm{w}}^{*}$, of wood beneath PU film were 9.92 and 14.45 , respectively, after 24-day irradiation (Table 2 and Fig. 3). In the PUS system $\Delta E_{\mathrm{f}}^{*}$ of PUS film reduced to 6.82, and $\Delta E_{\mathrm{w}}^{*}$, of wood beneath PUS film reduced to 9.26. Addition of Tinuvin-1130 into PU film evidently reduced discoloration of both film and underlying wood.

In the PUA- and PUAS-coated wood systems a slight discoloration of both films was observed during UV
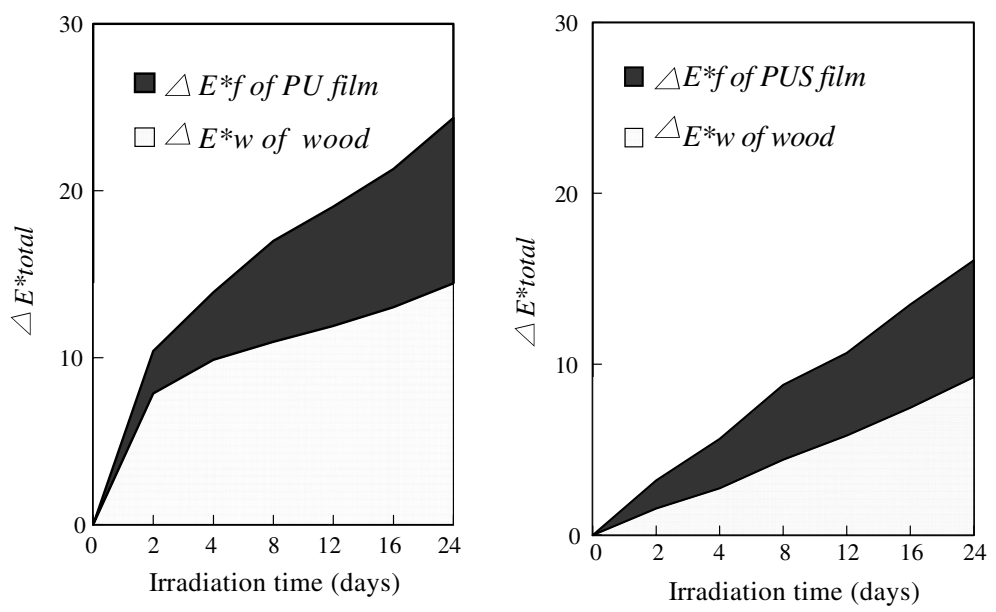

Fig. 3. Changes in $\Delta E_{\text {total }}^{*}$ of PU and PUS films and wood beneath films during irradiation. PU: aromatic polyurethane; PUS: PU with $2 \%$ Tinuvin-1130.
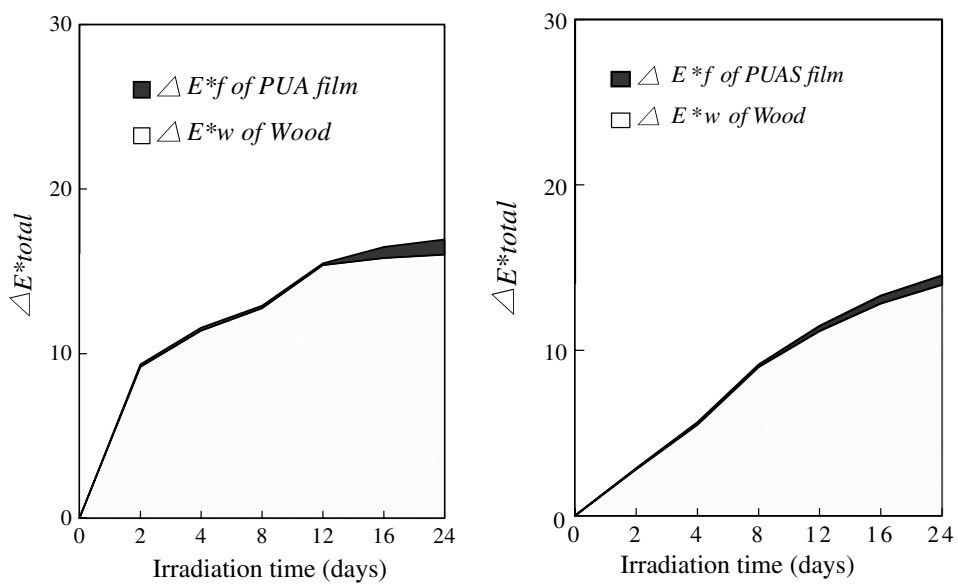

Fig. 4. Changes in $\Delta E_{\text {total }}^{*}$ of PUA and PUAS films and wood beneath films during irradiation. PUA: aliphatic polyurethane; PUAS: PUA with $2 \%$ Tinuvin-1130. 
irradiation (Fig. 4). $\Delta E_{\mathrm{w}}^{*}$ value of wood beneath PUA film increased significantly in the early stage of irradiation. After 24 days of UV irradiation $\Delta E_{\mathrm{f}}^{*}$ of PUA film and $\Delta E_{\mathrm{w}}^{*}$ of wood beneath PUA film were 0.89 and 16.34 , respectively, with corresponding $D_{\mathrm{f}}$ and $D_{\mathrm{w}}$ of $5.15 \%$ and $94.85 \%$. Discoloration of the PUA-coated system resulted mainly from the photoyellowing of the underlying wood. For the PUAS-coated system $\Delta E_{\mathrm{w}}^{*}$ value of specimens was less than 10 before 8 days of irradiation. However, $\Delta E_{\mathrm{w}}^{*}$ of wood beneath PUAS film increased to 14.19 after irradiated for 24 days. Comparing to $\Delta E_{\mathrm{w}}^{*}$ of wood beneath PUA film, it is clear that incorporating light stabilizer in PUA only reduced discoloration of the underlying wood in the early stage of UV irradiation.

\subsection{Effects of polyurethane coating on chemical characteristics of wood}

FTIR analysis was used to analyze chemical changes based on lignin absorption at $1510 \mathrm{~cm}^{-1}$ (Rodrigues et al., 1998; Pandey, 1999; Pandey and Pitman, 2004). Peak intensity at $1430 \mathrm{~cm}^{-1}(\mathrm{C}-\mathrm{H}$ in-plane deformation) was used as an internal reference.

The relative peak height ratio $\left(I_{1510} / I_{1430}\right)$ of control wood without coating decreased from 1.83 to 0.34 after 24 days of UV irradiation (Fig. 5), indicating lignin was severely degraded. There was a lesser decrease in the $I_{1510} /$ $I_{1430}$ ratio, from 1.83 to 0.80 , for the wood beneath PU film. The $I_{1510} / I_{1430}$ ratio of wood beneath PUS film decreased from 1.85 to 1.10 , indicating that addition of Tinuvin1130 into PU film was effective in protecting the wood from lignin degradation. Lignin degradation after 24-day irradiation occurred in the following order: wood beneath PUA

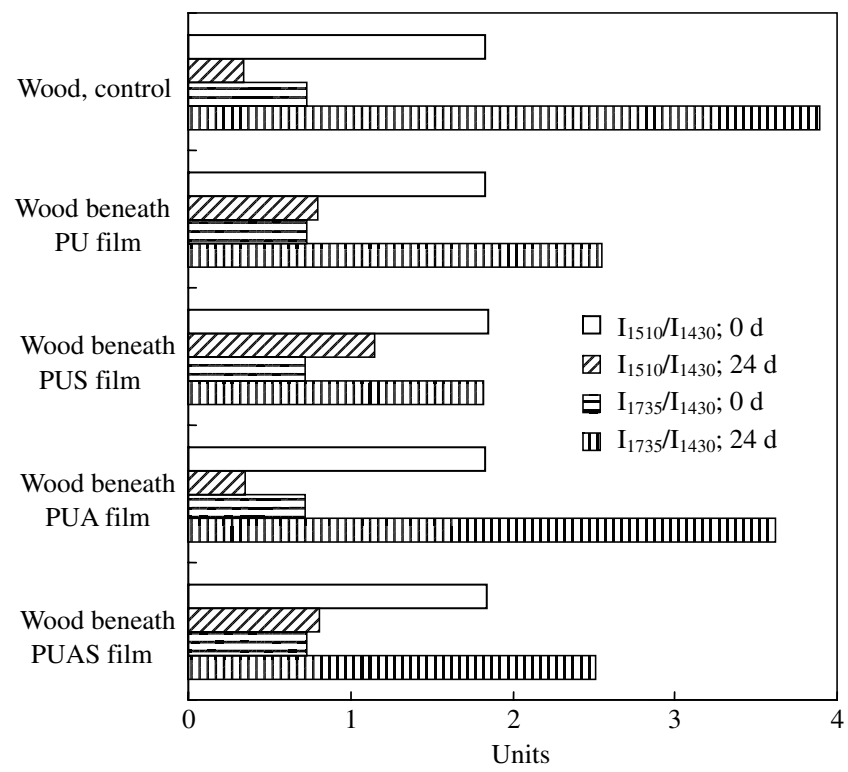

Fig. 5. Changes in $I_{1510} / I_{1430}$ and $I_{1735} / I_{1430}$ values of wood beneath various free films after 24 days of lightfastness test. PU: aromatic polyurethane; PUS: PU with 2\% Tinuvin-1130; PUA: aliphatic polyurethane; PUAS: PUA with $2 \%$ Tinuvin- 1130 . film $>$ wood beneath PU or PUAS films $>$ wood beneath PUS film. The lignin degradation of wood beneath PUA film was similar to that of control wood, indicating that PUA film was not able to protect the wood against lignin degradation but incorporating Tinuvin-1130 (PUAS) did provide some degree of protection. These results can be explained by the transmittance UV spectra of the free films (Fig. 2). Since PUA film transmits ultraviolet light of shorter wavelength (ca. $\lambda>250 \mathrm{~nm}$ ) and PUS film only transmits light (ca. $\lambda>370 \mathrm{~nm}$ ), PUS film is more effective than PUA film to protect the underlying wood from UV degradation of lignin.

The absorption at $1735 \mathrm{~cm}^{-1}$ is assigned to carbonyl groups $(\mathrm{C}=\mathrm{O})$, including nonconjugated carboxylic acid and ester. As shown in Fig. 5, the $I_{1735} / I_{1430}$ of un-coated wood increased from 0.72 to 3.89 after 24 days of UV irradiation and the wood beneath PUA film increased from 0.72 to 3.62 , indicating that the carbonyl groups were generated in almost equally amounts on both wood surfaces. The $I_{1735} / I_{1430}$ ratios of wood beneath PU and PUAS film were 2.55 and 2.51 , respectively. Increase in $I_{1735} / I_{1430}$ ratio of wood beneath the PUS film, from 0.72 to 1.82 , was the least among all test samples, indicating that PUS film was effective in alleviating generation of carbonyl derivatives of the underlying wood.

\section{Conclusions}

Wood is susceptible to photodiscoloration even it is coated with PU (photoyellowing type polyurethane) or PUA (non-photoyellowing type polyurethane) coatings. In this study, wood specimens covered with different free films were used to simulate clear-coated wood system. A light reflection model was established to quantify discoloration caused by the coating films and underlying wood separately. UV transmittance of PU film decreased while that of PUA film increased after lightfastness test. Moreover, incorporating light stabilizer into coatings decreased both photoyellowing and photodegradation. These results revealed that increased UV cutoff efficacy of clear coatings alleviated photoyellowing and photodegradation of clearcoated wood to extend service life of wood material. Use of the light reflection model provided further understanding of photodiscoloration caused by clear film and wood separately, and such information may be useful for designing novel wood clear coatings.

\section{References}

Barnes, H.M., Amburgey, T.L., Sanders, M.G., 2005. Performance of copper naphthenate and its analogs as ground contact wood preservatives. Bioresour. Technol. 96, 1131-1135.

Buckle, J.V., Acker, J.V., Stevens, M., 2005. Image processing as a tool for assessment and analysis of blue stain discoloration of coated wood. Int. Biodeter. Biodegr. 56, 178-187.

Chang, S.T., Chou, P.L., 1999. Photo-discoloration of UV-curable acrylic coatings and the underlying wood. Polym. Degrad. Stab. 63, 435-439. 
Chang, H.T., Chang, S.T., 2006. Modification of wood with isopropyl glycidyl ether and its effects on decay resistance and light stability. Bioresour. Technol. 97, 1265-1271.

Deka, M., Saikia, C.N., 2000. Chemical modification of wood with thermosetting resin: effect on dimensional stability and strength property. Bioresour. Technol. 73, 179-181.

Fiori, D.E., 1997. Two-component water reducible polyurethane coatings. Prog. Org. Coat. 32, 65-71.

Gindl, W., Zargar-Yaghubi, F., Wimmer, R., 2003. Impregnation of softwood cell walls with melamine-formaldehyde resin. Bioresour. Technol. 87, 325-330.

Hayoz, P., Wolfgang, P., Rogez, D., 2003. A new innovative stabilization method for the protection of natural wood. Prog. Org. Coat. 48, 297 309.

Hon, D.N.S., 2001. Weathering and photochemistry of wood. In: Hon, D.N.S., Shiraishi, N. (Eds.), Wood and Cellulosic Chemistry. Marcel Dekker, New York, pp. 513-571.

Kim, B.K., Pail, S.H., 1999. UV-curable poly(ethylene glycol)-based polyurethane acrylate hydrogel. J. Polym. Sci. Part A 37, 2703-2709.

Kultys, A., Pikus, S., 2001. Polyurethanes containing sulfur. III. New thermoplastic HDI-based segmented polyurethanes with diphenylmethane unit in their structure. J. Appl. Polym. Sci. Part A 39, 1733-1742.

Lai, Y.C., Quinn, E.T., 1995. Synthesis and characterization of UVcurable polysiloxane-based polyurethane prepolymer. J. Polym. Sci. Part A 33, 1783-1793.
Marcus, R.T., 1998. The measurement of color. In: Nassau, K. (Ed.), Color for Science, Art and Technology. Elsevier Science, Netherlands, pp. 31-91.

Pandey, K.K., 1999. A study of chemical structure of soft and hardwood and wood polymers by FTIR spectroscopy. J. Appl. Polym. Sci. 71, 1969-1975.

Pandey, K.K., Pitman, A., 2004. Examination of the lignin content in a softwood and a hardwood decayed by a brown rot fungus with the acetyl bromide method and Fourier transform infrared spectroscopy. J. Polym. Sci. Part A: Polym. Chem. 42, 2340-2346.

Pastore, T.C.M., Santos, K.O., Rubim, J.C., 2004. A spectrocolorimetric study on the effect of ultraviolet irradiation of four tropical hardwoods. Bioresour. Technol. 93, 37-42.

Rodrigues, J., Faix, O., Pereira, H., 1998. Determination of lignin content of Eucalyptus globules wood using FTIR spectroscopy. Holzforschung $52,46-50$.

Schwalm, R., Häupling, L., Reich, W., Beck, E., Enenkel, P., Menzel, K., 1997. Tuning the mechanical properties of UV coatings towards hard and flexible system. Prog. Org. Coat. 32, 191-196.

Sigh, R.P., Tomer, N.S., Bhadraiah, S.V., 2001. Photo-oxidation studies on polyurethane coating: effect of additives on yellowing of polyurethane. Polym. Degrad. Stab. 73, 443-446.

Williams, R.S., 2005. Weathering of wood. In: Rowell, R.M. (Ed.), Wood Chemistry and Wood Composite. CRC Press, USA, pp. 139185. 